\title{
MODULATION OF OSTEOBLAST CELL RESPONSE THROUGH LASER SURFACE PROCESSING OF NYLON 6,6
}

\author{
Paper (M1302) \\ David Waugh and Jonathan Lawrence \\ School of Engineering, University of Lincoln, Brayford Pool, Lincoln, Lincolnshire, LN6 7TS, UK.
}

\begin{abstract}
With an ageing population demand on medical facilities is growing, especially for bio-implants. Therefore, there is a need for cheaper, more efficient implants. This paper details how $\mathrm{CO}_{2}$ and $\mathrm{KrF}$ excimer lasers can be employed to modulate osteoblast cell growth on nylon 6,6 in relation to laser-modified wettability characteristics. Through patterning the contact angle, $\theta$, increased by up to $19^{\circ}$, indicating the presence of a mixed state wetting regime; whereas $\theta$ decreased by up to $20^{\circ}$ for the whole area irradiative processed samples. After 24 hours and 4 days incubation the cell cover density and cell count was somewhat modulated over the laser-modified samples compared to the as-received sample. A likely increase in surface toxicity gave rise to a hindered cell response for those samples with high energy densities and high incident pulse numbers. No strong correlations were determined for the laser-induced patterned samples which can be attributed to the likely mixed-state wetting regime. Correlative trends were found between the cell response, $\theta$, polar component and surface oxygen content for the whole area irradiative processed samples. Thus, allowing one to identify the potential for this technology in regenerative medicine.
\end{abstract}

\section{Introduction}

With numerous advances in medicine it has been seen that the population is ageing both within the U.K. and within other major countries around the world. On account of people living longer, a number of institutions have developed a focus on bioengineering to meet the ever growing demands on medical facilities (1). Furthermore, it is possible for one to realize that with an ageing population there is an ever increasing demand for biological implants. As a result, these needs of the ageing population need to be met more economically and efficiently so that costs and the need for unnecessary surgery are considerably reduced. Therefore, it is imperative for the biomedical industry to devise a way to manufacture cheap implants which can be used in confidence to ensure a dramatic reduction in failure rates. This can be met by the laser surface treatment of polymeric materials to enhance their biomimetic properties.

It has been realized worldwide within the scientific community and various industries that lasers offer major advantages over alternative techniques for materials processing (2-5). Some of the main advantages of using a laser for materials processing are:

- Relative cleanliness.

- Accurate processing.

- Precise placement of the beam onto the target material.

- Post-processing techniques required are usually minimal.

- Non-contact processing.

- Automation of a laser system is relatively easy to implement.

With a large number of different lasers now commercially available it is possible for one to deduce that almost all materials can be processed using a laser due to the wide range of laser parameters that can be utilized. With the many benefits of using lasers for materials processing it has been found that the interest in laser-induced surface treatment has grown, especially within the biomedical industry. This is due to the fact that lasers offer the user a highly selective, rapid technique to induce surface modification in both organic and inorganic materials (6).

In order to modify the surface properties of polymers for use in biological environments and enhance the cell response by improving upon the adhesion characteristics, a vast number of techniques and methods have been developed. These techniques range from surface topography modification (7-9) to surface chemistry modification (10-12) and have given rise to the increased interest in using polymers as biomaterials (13-20). Nylon 6,6, the strongest and most abrasive resistant unreinforced nylon, has been used for such biological applications as sutures, tracheal tubes and gastrointestinal segments (21). With regards to orthopedic applications it can be seen that nylon is not 
commonly employed due to the hygroscopic nature having a large affect on the mechanical properties over long periods of time (22). Having said that, the use of nylon 6,6 within this work gives high value experimentally insofar as to ascertain generic factors for polymeric materials which could be used to predict the osteoblast cell response. Also, by modifying the surface of polymeric materials it may be possible to identify other biological applications as this may enhance the osteoblast cell response and biocompatibile properties. On account of nylon 6,6 being a relatively inexpensive polymer when compared to other polymer types, by identifying other applications for this material the biological industry would benefit by being able to implement cheaper, more economic bio-implant materials.

On account of the many benefits offered by laser materials processing to life sciences this paper is a contribution to the endeavour of enhancing polymer surface properties to determine generic parameters which give rise to the level of biofunctionality. That is, this work undertakes an approach by which the laser modified surface parameters, wettability characteristics and osteoblast cell response are discussed in order to ascertain the variables which link the factors together as shown in Figure 1.

\section{Experimental Technique}

\section{Nylon 6,6 Material}

The nylon $6,6\left(\mathrm{~T}_{\mathrm{m}}: 255^{\circ}, \rho: 1.3 \mathrm{gcm}^{-3}\right)$ was sourced in $100 \mathrm{~mm}^{2}$ sheets with a thickness of $5 \mathrm{~mm}$ (Goodfellow Cambridge, Ltd). To obtain a conveniently sized sample for experimentation the as-received nylon sheet was cut into $20 \mathrm{~mm}$ diameter discs using a $1 \mathrm{~kW}$ continuous wave (cw) $\mathrm{CO}_{2}$ laser (Everlase S48; Coherent, Ltd).

\section{$\mathrm{CO}_{2}$ Laser-Induced Patterning}

In order to generate the required marking pattern with the Synrad $10 \mathrm{~W} \mathrm{CO}_{2}$ laser system, Synrad Winmark software version 2.1.0, build 3468 was used. The software was capable of using images saved as .dxf files which can be produced by using CAD programs such as, in this case, Licom AutoCaM. The nylon 6,6 samples were placed into the laser system onto a stage in which they were held in place using a bracket with a $20.5 \mathrm{~mm}$ diameter hole cut into the centre of the bracket. The surface of the sample was set to be 250 $\mathrm{mm}$ away from the output facet of the laser system to obtain focus and the system employed a galvanometer scanner to scan the $95 \mu \mathrm{m}$ spot size beam directly across the stationary target material. It should be noted that the target material and laser system was held in a laser safety cabinet in which the ambient gas was air and an extraction system was used to remove any fumes produced during laser processing.

There were four patterns induced onto the surfaces of the nylon 6,6 samples; were trenches with $50 \mu \mathrm{m}$ spacing (CT50), hatch with $50 \mu \mathrm{m}$ spacing (CH50), trenches with $100 \mu \mathrm{m}$ spacing (CT50) and hatch with $100 \mu \mathrm{m}$ spacing (CH100). In addition, an as-received control sample was used (AR). For each of the irradiated patterns the laser power was set to $70 \%$ (7 W) operating at $600 \mathrm{mms}^{-1}$.

\section{$\mathrm{CO}_{2}$ Laser Whole Area Irradiative Processing}

A cw $100 \mathrm{~W} \mathrm{CO}_{2}$ laser (DLC; Spectron, Ltd) was used to scan a $5 \mathrm{~mm}$ diameter beam across the target sample with one pass in order to irradiate the test area with an irradiance of $510 \mathrm{Wcm}^{2}$. By using a galvanometer, scanning speeds of $150,100,75,50,25$ and $20 \mathrm{mms}^{-1}$ were employed to irradiate six samples with effective fluences of 16.84 (samples CWA17), 25.51 (sample CWA26), 34.18 ( sample CWA34), 51.02 (sample CWA51), 102.04 (sample CWA102) and 127.55 (sample CWA128) $\mathrm{Jcm}^{-2}$, respectively. As with the laser-induced patterning experimentation the samples were held in place on a $\mathrm{z}$-variable stage with the whole experiment being carried out in a laser safety cabinet with the ambient gas being air.

\section{KrF Excimer Laser-Induced Patterning}

For the patterned experiments the repetition rate was kept constant at $25 \mathrm{~Hz}$, with a number of 10 pulses per site and used Aerotech CNC programming to induce the required pattern. A constant laser energy of $80 \pm 7$ $\mathrm{mJ}$ was used with the attenuator set to $0.3(30 \%)$ giving a measured energy at the target sample of $23.67 \pm 2.5 \mathrm{~mJ}$, resulting in a fluence of $858 \pm 91$ $\mathrm{mJ} / \mathrm{cm}^{2}$. In order to induce the intended pattern a projection imaging system was implemented with a focusing lens of x10 demagnification. The patterns induced using this technique were $50 \mu \mathrm{m}$ trench (ET50), $100 \mu \mathrm{m}$ trench (ET100), $50 \mu \mathrm{m}$ hatch (EH50) and $100 \mu \mathrm{m}$ (EH100). Two non-contact masks were used for both dimensioned patterns which included a brass mask with six $1 \mathrm{~mm}$ diameter holes spaced by 2 $\mathrm{mm}$, centre to centre, for the $100 \mu \mathrm{m}$ dimensions and a SS316 foil (Laser Micromachining Ltd., UK) with five $0.5 \mathrm{~mm}$ diameter holes spaced by $1.5 \mathrm{~mm}$, 'centre to centre', for the $50 \mu \mathrm{m}$ dimensions. To keep the constant 10 pulses per site it should also be noted here that scanning velocities of $0.125 \mathrm{mms}^{-1}$ and $0.25 \mathrm{mms}^{-1}$ were used for the $50 \mu \mathrm{m}$ and $100 \mu \mathrm{m}$ dimensioned patterns, respectively. 
No processing gases were used throughout the experimentation and all laser processing was carried out in an enclosure in which the ambient gas was air. Also, for all laser processing no homogonizer was implemented meaning that the raw beam was used which would have given rise to energy spikes pulse to pulse, having some possible effect on the incident laser fluence and laser material processing.

\section{KrF Excimer Laser Whole Area Irradiative Processing}

For the whole area processing with an excimer laser the raw $23 \times 12 \mathrm{~mm}^{2}$ beam was used to irradiate a large section of each sample at a time. In order to hold the sample normal to the beam a bracket on the optical train was used. For the large area processing experiments 6 samples where studied; these being 100 pulses at $100 \mathrm{~mJ}$ (EWA100), 100 pulses at $150 \mathrm{~mJ}$ (EWA150), 100 pulses at $200 \mathrm{~mJ}$ (EWA200), 100 pulses at $250 \mathrm{~mJ}$ (EWA250), 500 pulses at $250 \mathrm{~mJ}$ (EWA250_500) and 1000 pulses at $250 \mathrm{~mJ}$ (EWA250_1000). This gave fluences of $36 \pm 3,54 \pm 5$, $72 \pm 8$ and $\overline{9} 1 \pm 10 \mathrm{mJcm}^{-2}$, respectively for the different energies used. Throughout the whole area excimer experiments the repetition rate was kept constant at 25 $\mathrm{Hz}$ and Aerotech $\mathrm{CNC}$ programming ensured that the correct number of pulses was applied to each sample.

\section{Topography, Wettability Characteristics and Surface Chemistry Analysis}

After laser irradiation the nylon 6,6 samples were analysed using a number of techniques. The surface profiles were determined using a white light interferometer (WLI) (NewView 500; Zygo, Ltd) with MetroPro and TalyMap Gold Software. The WLI was set-up using a $\times 10$ Mirau lens with a zoom of $\times 0.5$ and working distance of $7.6 \mathrm{~mm}$. This system also allowed $\mathrm{Sa}$ and $\mathrm{Ra}$ roughness parameters to be determined for each sample.

In accordance with the procedure detailed by Rance (23) the samples were ultrasonically cleaned in isoproponal (Fisher Scientific Ltd.) for 3 minutes at room temperature before using a sessile drop device to determine various wettability characteristics. This was to allow for a relatively clean surface prior to any $\theta$ measurements being taken. To ensure that the sample surfaces were dry a specimen dryer (Metaserv, Ltd.) was employed to blow ambient air across the samples. A sessile drop device (OCA20; Dataphysics Instruments, $\mathrm{GmbH}$ ) was used with relevant software (SCA20; Dataphysics Intrsuments, $\mathrm{GmbH}$ ) to allow the recent advancing and receding $\theta$ for triply distilled water and the recent advancing angle for diiodomethane to be determined for each sample.
Thereafter the advancing $\theta$ for the two liquids were used by the software to draw an OWRK plot to determine the surface energy of the samples. For the two reference liquids the SCA20 software used the Ström et al technique (triply distilled water SFT(total:72.80), SFT(D:21.80), SFT(P:51.00); diiodomethane - SFT(total:50.80), SFT(D:50.80), SFT(P:0.00)) to calculate the surface energy of the material. It should be noted here that ten $\theta$, using two droplets in each instance, were recorded to achieve a mean $\theta$ for each liquid and surface.

All samples were analysed using x-ray photoelectron spectroscopy (XPS). This allowed any surface modifications in terms of surface oxygen content due to the laser irradiation to be revealed. XPS measurements were performed on a photoelectron spectrometer (Axis Ultra DLD; Kratos Analytical Ltd., UK) employing monochromatic aluminium k-alpha radiation source, operating at $120 \mathrm{~W}$ power and an associated photon energy of $1486.6 \mathrm{eV}$. To test the reproducability of the surface, two sections of each sample were analysed; the analysis area on each sample was $700 \times 300$ microns. The spectrometer was run in its Hybrid mode and spectra were acquired at pass energies of $20 \mathrm{eV}$ (for the high resolution scans) and $160 \mathrm{eV}$ for the survey scans. All data was analysed through CasaXPS (v2.3.14) analysis software using sensitivity factors supplied by the instrument manufacturer.

\section{In Vitro Experimentation}

Prior to any biological testing being carried out the samples were autoclaved (D-Series Bench-Top Autoclave; Systec, GmbH) to ensure that all samples were sterilized. For all biological work undertaken, unless stated, a biological safety cabinet (BSC) (Microflow Class II ABS Cabinet; BioQuell UK, Ltd) was used to create a safe working environment and to provide a clean, sterile environment to manipulate the cells used.

Normal human osteoblast cells (Clonetics CC-2538; Lonza, Inc.) were initially cultured in a T75 $(75 \mathrm{ml})$ flask by suspending the cells in $19 \mathrm{ml}$ culture medium comprising of $90 \%$ eagle minimum essential medium (Sigma-Aldrich, Ltd., UK) and 10\% foetal bovine serum (FBS) (Sigma-Aldrich Ltd., UK). The flask was then placed in an incubator and left for $24 \mathrm{hrs}$. After $24 \mathrm{hrs}$ the cells were assessed and the spent media was aspirated before dispensing $15 \mathrm{ml}$ of fresh media and returning the flask to the incubator for three days.

The period of three days allowed the cells to become confluent in the flask providing enough cells for seeding onto the samples. The cells were detached 
from the flask using $5 \mathrm{ml}$ Trypsin-EDTA (SigmaAldrich Ltd., UK) whilst placed in the incubator for seven minutes. Once all cells had become detached $10 \mathrm{ml}$ culture medium was added to neutralize the Trypsin. In order to aspirate the supernatant the cell culture was centrifuged (U-320R; Boeco, $\mathrm{GmbH}$ ) for five minutes at $200 \mathrm{~g}$. To ensure the cells were ready for seeding they were resuspended in $10 \mathrm{ml}$ of culture medium and dispensed between the samples in the 6well plates. This equated to $0.55 \mathrm{ml}\left(2 \times 10^{4}\right.$ cells $\left./ \mathrm{ml}\right)$ for each sample. The well plates were then placed in the incubator for a set time. One plate was removed after $24 \mathrm{hrs}$ and two other plates after four days. A well plate after 24 hrs and 4 days was prepared for the SEM as will be discussed later and the other plate, removed after 4 days, was prepared for counting using an improved neubauer hemacytometer (Fisher Scientific Ltd., UK) by mixing $10 \mu \mathrm{l}$ of each cell suspension with $10 \mu \mathrm{l}$ of trypan-blue (Sigma-Aldrich Ltd., UK). In order to harvest the cells for counting the cells were detached from the samples using $2 \mathrm{ml}$ Trypsin-EDTA (Sigma-Aldrich Ltd., UK) whilst placed in the incubator for seven minutes. Once all cells had become detached $4 \mathrm{ml}$ culture medium was added to neutralize the Trypsin. In order to aspirate the supernatant the cell culture was centrifuged (U-320R; Boeco, $\mathrm{GmbH}$ ) for five minutes at $200 \mathrm{~g}$. To ensure the cells were ready for counting they were resuspended in $2 \mathrm{ml}$ of culture medium and $2 \mathrm{ml}$ of the trypan-blue was added.

\section{Scanning Electron Microscopy and Cell Cover Density of In Vitro Samples}

In order to view the attached cells using SEM it was necessary to undertake a procedure to produce a sample that was dehydrated ready for Au coating. The samples were initially rinsed with phosphate-buffered saline (PBS) (Sigma-Aldrich, UK) to remove any unattached cells and then adherent cells were fixed using $1.2 \%$ glutaraldehyde in water (Sigma-Aldrich, UK) at room temperature for one hour within the BSC. After an hour the glutaraldehyde solution was removed and the fixed cells were washed with PBS prior to carrying out a graded series of ethanol/distilled water mixtures of 50/50, 80/20, 90/10, 95/5, 98/2 and 100/0. Each sample was left in these mixtures for $10 \mathrm{~min}$ to ensure dehydration. Once this procedure was carried out, the samples were mounted and sputter coated with $\mathrm{Au}$ so that SEM micrographs could be obtained. In order to produce the best images possible each image was manipulated in terms of brightness, contrast and gamma by using ImagePro Version 5.0.0.39 for Windows XP/Professional software (Media Cybernetics Inc., USA).
In addition to the cell count described in Section 3.5, the cell cover density was determined following both $24 \mathrm{hrs}$ and 4 day incubation. This was done by analysing the cell coverage on each sample using SEM and optical micrographs with the ImagePro software. The optical micrographs were obtained using an upright optical microscope (Flash 200 Smartscope; OGP, Ltd) with magnifications varying between $\times 20$ and $\times 100$.

\section{Results and Discussion}

\section{Topography $-\mathrm{CO}_{2}$ and $\mathrm{KrF}$ Excimer Laser Processing}

Topography modification through the different laser surface treatments has been discussed previously $(21,24)$.

It was evident that a $\mathrm{CO}_{2}$ laser can be employed to modify significantly the surface topography of nylon 6,6 if the laser-material interaction threshold in terms of fluence is exceeded. The $\mathrm{CO}_{2}$ laser whole area irradiated processed samples at large fluencies (sample CWA102 and sample CWA28) gave rise to an order of magnitude increase in Sa. It was also found that the topographies of the $\mathrm{CO}_{2}$ laser-induced patterned samples were somewhat periodic with steep, sharp gradients when considering the peak heights; whereas, the $\mathrm{CO}_{2}$ laser whole area irradiative processed samples gave rise to a more cratered surface. This was on account of more melting taking place which in turn gave rise to evolved gases being able to break to the surface. As a result of this, the maximum peak heights achieved for the $\mathrm{CO}_{2}$ laser whole area processed were found to be considerably larger by up to $13 \mu \mathrm{m}$ but were less densely situated when compared to the $\mathrm{CO}_{2}$ laser patterned samples which had maximum peaks heights of approximately 2 to $3 \mu \mathrm{m}$ and were more periodic.

For the $\mathrm{CO}_{2}$ whole area irradiative processed samples it was found that in terms of surface topography the first three samples (samples CWA17, CWA26 and CWA34) gave rise to negligible modifications when compared to the as-received sample (AR) while all of the $\mathrm{CO} 2$ laser-induced patterned samples gave rise to a noteworthy variation in the surface topography of the nylon 6,6. This can be on account of samples CWA17, CWA26 and CWA34 having been irradiated with fluences below or around the threshold fluence. On account of this, one can then deduce that all other samples gave rise to significant modifications in the surface topography as the fluences used to irradiate the other samples were considerably higher. As such, one can reasonably state that above the threshold, both $\mathrm{CO}_{2}$ 
whole area processing and laser-patterning gave rise to considerable modifications in terms of topography of the nylon 6,6 samples.

It has been observed that $\mathrm{KrF}$ excimer laser-induced patterning can significantly modify the topography of nylon 6,6 insofar as the roughness had increased and distinct periodic patterns had been induced into the material surface. In contrast, the $\mathrm{KrF}$ excimer whole area irradiative processed samples gave rise to a negligible variation in surface parameters when compared to the as-received sample such that the Sa remained around 0.1 to $0.2 \mu \mathrm{m}$ and $\mathrm{Ra}$ around 0.03 $\mu \mathrm{m}$.

\section{Wettability $-\mathrm{CO}_{2}$ and $\mathrm{KrF}$ Excimer Laser Processing}

The wettability results have been discussed in more depth previously $(21,24)$.

Owed to the fact that the $\mathrm{CO}_{2}$ laser-induced patterned samples induced a more hydrophobic response and a more hydrophilic response was observed for the $\mathrm{CO}_{2}$ whole area irradiated patterns it is possible to deduce that surface oxygen content may not be the main driving force for wettability as the oxygen content increased in all instances by up to $2 \%$ at. for the patterned samples and up to $5 \%$ at. for the whole area irradiated samples (see Table 1). This is owed to the thermolytical interaction between the nylon 6,6 material and the $\mathrm{CO} 2$ laser light that gives rise to melting of the nylon surface allowing oxidation to take place.

Upon analysing the $\mathrm{CO}_{2}$ laser-induced patterned surfaces it was found that the $\gamma^{T}$ and $\gamma^{\mathrm{P}}$ had decreased by up to $7 \mathrm{mJm}^{-2}$ and the surface roughness had considerably increased. It was found that $\gamma^{\mathrm{P}}$ and $\gamma^{\mathrm{T}}$ for the samples studied were both a decreasing function of the $\theta$, which correlates with current theory; however, current theory states that $\theta$ for a hydrophilic surface should decrease upon increasing surface roughness which has not been seen throughout this experimentation.

Table 2 allows one to identify that there was some significant differences between the as-received (AR), $\mathrm{KrF}$ excimer laser-induced patterned and $\mathrm{KrF}$ excimer laser whole area processed nylon 6,6 samples in terms of surface parameters and wettability characteristics.

Table 1 - Results summary for samples showing roughness parameters, surface oxygen content and wettability characteristics following CO2 laser processing of nylon 6,6 .

\begin{tabular}{|c|c|c|c|c|c|c|c|}
\hline $\begin{array}{c}\text { Sample } \\
\text { ID }\end{array}$ & $(\mu \mathrm{m})$ & $(\mu \mathrm{m})$ & $\begin{array}{c}\text { Polar } \\
\text { Component, } \gamma^{\mathrm{P}} \\
\left(\mathrm{mJm}^{-2}\right)\end{array}$ & $\begin{array}{c}\text { Dispersive } \\
\text { Component, } \gamma^{\mathrm{D}} \\
\left(\mathrm{mJm}^{-2}\right)\end{array}$ & $\begin{array}{c}\text { Total Surface } \\
\text { Energy, } \gamma^{\mathrm{T}} \\
\left(\mathrm{mJm}^{-2}\right)\end{array}$ & $\begin{array}{c}\text { Surface } \\
\text { Oxygen } \\
\text { Content } \\
\text { (\%at.) }\end{array}$ & $\begin{array}{c}\text { Contact } \\
\text { Angle } \\
\left({ }^{\circ}\right)\end{array}$ \\
\hline AR & 0.126 & 0.029 & 17.69 & 29.66 & 47.34 & 13.26 & $56.4 \pm 1.2$ \\
\hline \multicolumn{8}{|c|}{$\mathrm{CO}_{2}$ Laser-Induced Patterned Samples } \\
\hline CT50 & 0.636 & 0.148 & 12.24 & 28.63 & 40.87 & 14.33 & $66.0 \pm 4.0$ \\
\hline CT100 & 0.297 & 0.185 & 16.86 & 29.83 & 46.69 & 14.05 & $57.5 \pm 2.4$ \\
\hline $\mathrm{CH} 50$ & 0.423 & 0.103 & 10.93 & 31.64 & 42.58 & 14.99 & $65.8 \pm 2.9$ \\
\hline $\mathrm{CH} 100$ & 0.326 & 0.155 & 13.63 & 30.37 & 44.00 & 14.84 & $62.2 \pm 2.3$ \\
\hline \multicolumn{8}{|c|}{$\mathrm{CO}_{2}$ Whole Area Irradiative Processed Samples } \\
\hline CWA17 & 0.111 & 0.060 & 18.36 & 28.75 & 47.11 & 13.56 & $55.8 \pm 2.7$ \\
\hline CWA26 & 0.100 & 0.158 & 19.67 & 28.35 & 48.02 & 13.86 & $54.6 \pm 3.5$ \\
\hline CWA34 & 0.101 & 0.092 & 14.89 & 38.55 & 53.43 & 14.34 & $56.4 \pm 3.5$ \\
\hline CWA51 & 0.341 & 0.139 & 10.66 & 38.59 & 49.26 & 15.45 & $64.1 \pm 2.2$ \\
\hline CWA102 & 4.356 & 1.236 & 28.49 & 23.82 & 52.31 & 16.77 & $46.6 \pm 4.7$ \\
\hline CWA128 & 3.201 & 1.335 & 31.98 & 22.78 & 54.76 & 18.93 & $43.0 \pm 3.7$ \\
\hline
\end{tabular}


It was observed that for the $\mathrm{KrF}$ laser-induced patterned samples $\theta$ increased even though there was a significant increase in surface roughness. On the other hand, it was found that on account of an increase in $\gamma^{\mathrm{P}}$ and $\gamma^{T}, \theta$ decreased for the $\mathrm{KrF}$ excimer laser whole area irradiative processed nylon 6,6 samples. It was observed that for all $\mathrm{KrF}$ excimer laser processed samples the surface oxygen content increased by up to $5 \%$ at. when compared to the as-received sample (AR). This increase in surface oxygen content indicates that this may have given rise to the observed reduction in $\theta$ for the laser whole area processed samples. Still, this is does not seem to be the case as a reduction in $\theta$ was not observed for the $\mathrm{KrF}$ excimer laser-induced patterned samples.

This suggests that a significant variation in surface topography of the nylon 6,6 may bring about the change in wettability regime; whereas when there is no significant variation in topography it is possible that a variation in wettability is brought about by other parameters such as $\gamma^{\mathrm{P}}$ and $\gamma^{\mathrm{T}}$. In addition, even though there was a change in wetting regime for the $\mathrm{KrF}$ excimer laser-induced patterned samples to account for the increase of $\mathrm{Sa}$ and $\mathrm{Ra}$, it was still found that $\theta$ remained strongly linked to variations in $\gamma^{\mathrm{P}}$ and $\gamma^{\mathrm{T}}$.
In terms of collating the $\gamma^{\mathrm{P}}$ and $\gamma^{\mathrm{T}}$ results for the entire $\mathrm{KrF}$ excimer laser processed samples and the effects thereof on $\theta$, Figure 7.7 shows that there was a strong inverse function correlation between $\theta$ and the surface energy parameters regardless of the processing technique used. This indicates that $\gamma^{\mathrm{P}}$ and $\gamma^{\mathrm{T}}$ could be the main driving parameters determining the wettability of the nylon 6,6 samples in this instance. As a result it may be possible to use these parameters as indicators of how nylon 6,6 will wet as long as the results are repeatable. Having said that, as discussed in Section 7.3.2, similar values of $\gamma^{\mathrm{P}}$ and $\gamma^{\mathrm{T}}$ give rise to equivalent $\theta$ suggesting that this process is repeatable.

\section{Cell Cover Density and Cell Count}

The osteoblast cell response, in terms of morphology, has been discussed in more depth previously $(24,25)$.

Figure 1 allows one to see that overall the laser surface treated samples did not have a correlative trend between the cell cover density, cell count and $\theta$. This is on account of the laser-induced patterned samples not correlating with the trend observed for the laser whole area irradiative processed samples which can be attributed to the transition in wetting regime for the laser-induced patterned samples giving erroneous results.

Table 2 - Results summary for samples showing roughness parameters, surface oxygen content and wettability characteristics for $\mathrm{KrF}$ processed nylon 6,6.

\begin{tabular}{|c|c|c|c|c|c|c|c|}
\hline Sample ID & $\begin{array}{c}\mathrm{Sa} \\
(\mu \mathrm{m})\end{array}$ & $\begin{array}{c}\mathrm{Ra} \\
(\mu \mathrm{m})\end{array}$ & $\begin{array}{c}\text { Polar } \\
\text { Component, } \gamma^{\mathrm{P}} \\
\left(\mathrm{mJm}^{-2}\right)\end{array}$ & $\begin{array}{c}\text { Dispersive } \\
\text { Component, } \gamma^{\mathrm{D}} \\
\left(\mathrm{mJm}^{-2}\right)\end{array}$ & $\begin{array}{c}\text { Total Surface } \\
\text { Free Energy, } \gamma^{\mathrm{T}} \\
\quad\left(\mathrm{mJm}^{-2}\right)\end{array}$ & $\begin{array}{c}\text { Surface } \\
\text { Oxygen } \\
\text { Content } \\
\text { (\%at.) }\end{array}$ & $\begin{array}{l}\text { Contact } \\
\text { Angle } \\
\left(^{\circ}\right)\end{array}$ \\
\hline AR & 0.126 & 0.029 & 17.69 & 29.66 & 47.34 & 13.26 & $56.4 \pm 1.2$ \\
\hline \multicolumn{8}{|c|}{ Laser-Induced Patterned Samples } \\
\hline ET50 & 0.281 & 0.312 & 15.48 & 28.74 & 44.22 & 13.87 & $60.6 \pm 3.0$ \\
\hline ET100 & 1.320 & 0.679 & 6.52 & 23.54 & 30.06 & 13.53 & $80.8 \pm 5.2$ \\
\hline EH50 & 0.739 & 0.548 & 12.94 & 17.24 & 30.17 & 14.15 & $75.1 \pm 3.9$ \\
\hline EH100 & 1.530 & 1.032 & 17.06 & 13.55 & 30.61 & 14.83 & $73.0 \pm 3.7$ \\
\hline \multicolumn{8}{|c|}{ Whole Area Irradiative Processed Samples } \\
\hline EWA100 & 0.119 & 0.029 & 21.72 & 27.08 & 48.79 & 13.11 & $52.8 \pm 1.1$ \\
\hline EWA150 & 0.092 & 0.039 & 25.98 & 24.76 & 50.74 & 13.03 & $49.1 \pm 4.7$ \\
\hline EWA200 & 0.108 & 0.030 & 25.26 & 25.86 & 51.12 & 13.28 & $48.9 \pm 2.6$ \\
\hline EWA250 & 0.107 & 0.031 & 22.37 & 28.66 & 51.03 & 13.24 & $50.3 \pm 1.5$ \\
\hline EWA250_500 & 0.164 & 0.039 & 33.31 & 21.40 & 54.71 & 15.32 & $42.9 \pm 1.6$ \\
\hline EWA250_1000 & 0.198 & 0.036 & 37.56 & 20.89 & 58.46 & 18.36 & $37.6 \pm 3.2$ \\
\hline
\end{tabular}


For the laser whole area irradiative processed samples, with the exception of sample CWA102 and sample CWA128, the cell cover density and cell count were an increasing function of $\theta$. It should be noted here that sample CWA102 and sample CWA128 did not follow the trend on account of the toxicity dominating.

Figure 2 shows that there was no correlative relationship between $\gamma^{\mathrm{P}}, \gamma^{\mathrm{T}}$ and osteoblast cell response for the laser surface treated nylon 6,6 samples when taken as a whole. Similar to $\theta$ (see Figure 1), this can be accounted for by the laser-induced patterned samples which gave rise to somewhat erroneous results on account of the likely transition in wetting regime. The relationship between cell cover density, cell count and $\gamma^{\mathrm{P}}$ for the entire laser whole area irradiative processed samples can be seen in Figure 2 in that the enhancement of osteoblast cell response was an increasing function of $\gamma^{\mathrm{P}}$. However, sample CWA102 and sample CWA128 did not correspond with the trend identified and can be explained by the increase in surface toxicity. Leading on, it can be seen from Figure 2 that for none of the samples there did not appear to be any correlative relationship between $\gamma^{\mathrm{T}}$ and the osteoblast cell response, indicating that $\gamma^{\mathrm{T}}$ was not a dominant parameter. Even though there was no overall trend observed for $\theta$ and $\gamma^{\mathrm{P}}$ it can still be said within reason that if a surface toxicity was low enough as to not dominate the osteoblast cell response, then it appears likely that $\theta$ and $\gamma^{\mathrm{P}}$ play an important role in determining the osteoblast cell response to the laser whole area irradiative processed nylon 6,6.

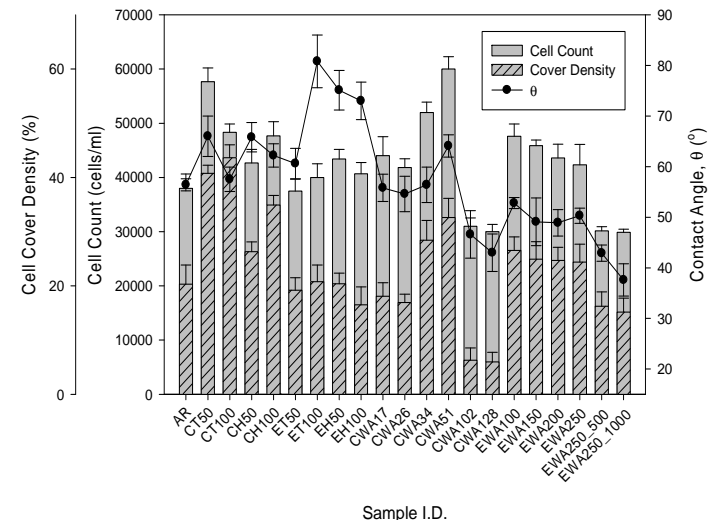

Figure 1 - Histogram showing cell count and cell cover density in relation to $\theta$.

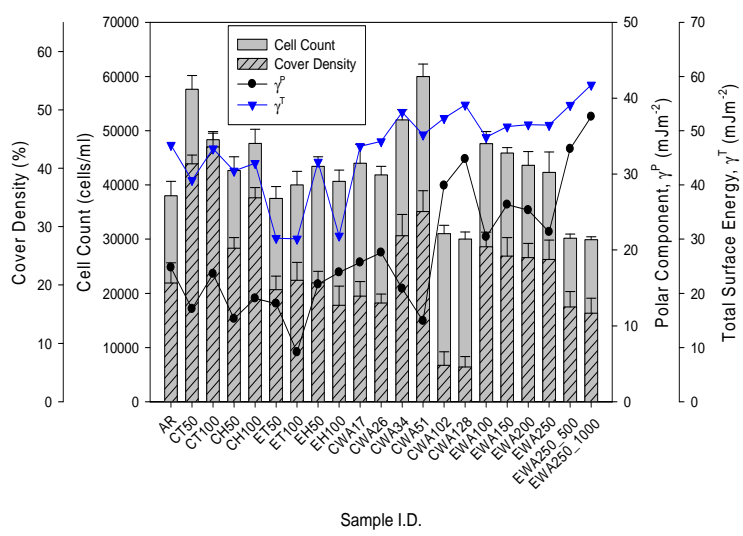

Figure 2 - Histogram showing cell count and cell cover density in relation to $\gamma^{\mathrm{P}}$ and $\gamma^{\mathrm{T}}$.

It can be seen from Figure 3 that overall, the surface roughness in terms of $\mathrm{Ra}$ and Sa did not appear to have a distinct relationship with the osteoblast cell response with regards to cell cover density and cell count. Having said that, for those samples with the largest values of Sa and Ra (sample CWA102 and sample CWA128) the surface roughness could be indirectly indicative of the bioactive nature of the $\mathrm{CO}_{2}$ laser whole area irradiative processed nylon 6,6 samples. This is due to the fact that the melting which gave rise to the proposed increase in toxicity, which dominated the osteoblast cell response, also gave rise to the significant increase in Ra and Sa.

When taking into account all samples with respect to the relationship between the surface oxygen content and osteoblast cell response, it can be seen from Figure 4 that there was no overall trend which could be implemented to predict the osteoblast cell response to the laser surface treated nylon 6,6. Having said that, there was two different observed trends for the laser whole area irradiative processed samples which could potentially be used as an indirect method to estimate whether enhanced osteoblast cell response can be brought about by the nylon 6,6 samples. This is accounted for by the likelihood of the nylon 6,6 samples becoming more toxic upon more laser-induced melting even though the surface will also oxidize, giving rise to an increase in surface oxygen content. 


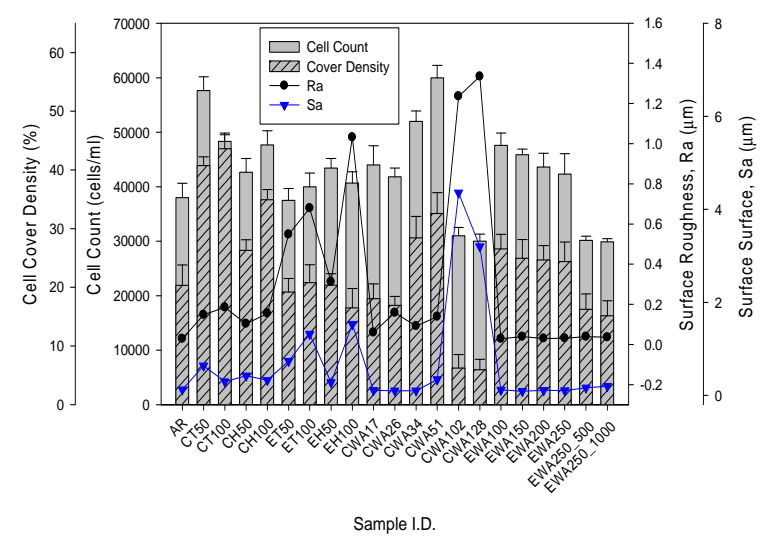

Figure 3 - (a) Histogram showing cell count and cell cover density in relation to $\mathrm{Ra}$ and $\mathrm{Sa}$.

In terms of cell cover density and cell count it has been seen that below a potential toxicity threshold $\theta$ and $\gamma^{\mathrm{P}}$ appear to dominate the bioactive nature of nylon 6,6 which has undergone laser whole area irradiative processing regardless of the laser type used. With this in mind, one can extrapolate that $\theta$ and $\gamma^{\mathrm{P}}$ can be implemented to predict how osteoblast cells will react upon laser whole area irradiative processed nylon 6,6.

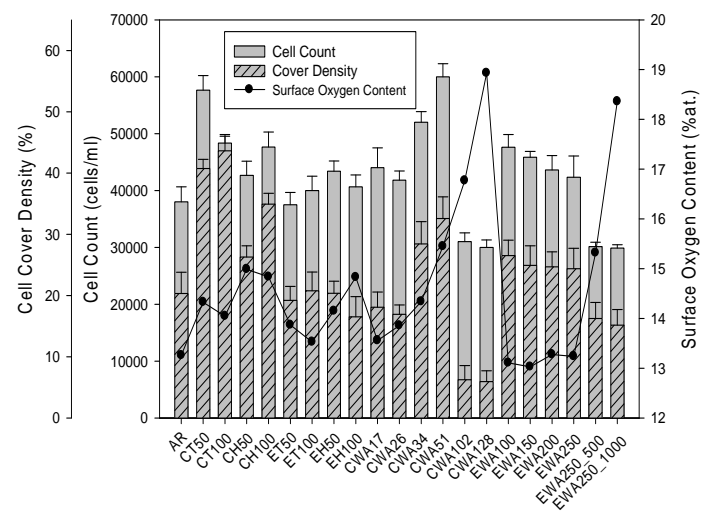

Sample I.D.

Figure 4 - (a) Histogram showing cell count and cell cover density in relation to surface oxygen content.

\section{Conclusions}

Throughout this research it has been further demonstrated that lasers offer an efficient and convenient means of surface modifying materials - in this case nylon 6,6, both chemically and topographically.

Upon analysing all of the laser processed surfaces it was determined that a number of surface parameters can be modified. These surface modifications brought about variations in the wettability characteristics of the nylon 6,6 samples. It was found that total surface energy, $\gamma^{\mathrm{T}}$, and polar component, $\gamma^{\mathrm{P}}$, had decreased by along with an increase in surface roughness. It was also determined that $\gamma^{\mathrm{P}}$ and $\gamma^{\mathrm{T}}$ for the samples studied were both a decreasing function of $\theta$, which correlated with current theory; however, current theory also states that $\theta$ for a hydrophilic surface should decrease upon increasing surface roughness which has not been seen throughout this experimentation. This can be attributed to an intermediate mixed Cassie-Baxter/Wenzel regime, in which both Wenzel and Cassie-Baxter regimes arise at the solid-liquid interface as a result of the formation of the water droplet on the various laser modified surface topographies.

With regards to the bioactive nature of the nylon 6,6 samples, the laser-induced patterned samples did not give rise to any identifiable trends between the lasermodified surface parameters and the cell response. This was attributed to the mixed-state wetting regime having a large influence on cell response. On the other hand, it was found for the laser whole area processed samples that $\theta$ and $\gamma^{\mathrm{P}}$ had strong trends with the osteoblast cell count and cover density. On account of this, one can see that laser whole area processing, regardless of the wavelength, offers a convenient means to tailor materials to enhance osteoblast cell growth.

\section{References}

(1) Bauerle D. Laser Processing and Chemistry. Third Ed. New York, USA: Springer-Verlag; 2000.

(2) Adamson AW, Gast AP. Physical Chemistry of Surfaces. Sixth ed. New York, USA: John Wiley \& Sons Inc.; 1997.

(3) Adam N.K., Livingston H.K. (1958) Contact angles and the work of adhesion. Nature 182128.

(4) Boehlen K.L., Stassen-Boehlen I., Allot R. Advanced Laser Micro-Structuring of Super Large Area Optical Films. Exitech Ltd Online Articles and Papers :07 November 2007.

(5) Adam NK, Elliott GEP. (1958) The effects of high temperature variations on contact angle measurements of vitreous enamels. J. Chem. Soc. 182206.

(6) Adamson A.W. (1973) Potential Distortion Model for Contact Angle and Spreading II. Temperature Dependant Effects. J. Coll. Interf. Sci. 44273. 
(7) Botero AECea. Surface chemistry fundamentals of biosorption of Rhodococcus opacus and its effect in calcite and magnesite flotation. Minerals Engineering 2008;21:83.

(8) Broderick N.G.R. (1999) Large mode area fibers for high power applications. Opt. Fiber Technol. 5 185-196.

(9) Burton Z, Bhushan B. (2005) Hydrophobicity, adhesion and friction properties of nanopatterned polymers and scale dependence for micro- and nanoelectromechanical systems. Nano Letters 5 16071613.

(10) Chu PK. (2006) Bioactivity of plasma implanted biomaterials. Nucl. Inst. Meth. Phys. Res. B. 242 1-7.

(11) Ma Z, Mao Z, Gao C. (2007) Surface modification and property analysis of biomedical polymers used for tissue engineering. Coll. Surf. B. 60 137-157.

(12) Chu P.K. (2006) Plasma surface treatment of artificial orthopedic and cardiovascular biomaterials. Surf. Coat. Technol. 201 5601-5606.

(13) Jung, Y.C., Bhushan, B. (2006) Contact angle, adhesion and friction properties of micro- and nanopatterned polymers for superhydrophobicity. Nanotechnol. 17 4970-4980.

(14) Neumann AW. (1974) Contact Angles:

Thermodynamic Status, Measurement, Interpretation and Application. Adv. Coll. Int. Sci. 4105.

(15) Carew E.O. Chapter 1 - Properties of Materials. In: Ratner BDea, editor. Biomaterials Science. Second ed. San Diego, California, USA: Elsevier Academic Press; 2004. p. 23.

(16) Lippert T., Wei J., Wokaun A., Hoogen N., Nuyken O. (2000) Polymers designed for laser microstructuring. Appl. Surf. Sci. 168 270-272.

(17) Hao L, Lawrence J. Low D.K.Y., Lim G.C., Zheng H.Y. (2004) Correlation of the hyrdroxyl bond and wettability characteristics of a magnesia partially stabilised zirconia following $\mathrm{CO}_{2}$ laser irradiation. Thin Solid Films 2004468 12-18.

(18) Mirzadeh H., Katbab A.A., Khorsani M.T., Burford R.P., Gorgin E., Golestani A. (1995) Cell attachment to laser-induced AAm-and HEMA-grafted ethylene-propylene rubber as biomaterial: in vivo study. Biomaterials 16 641-648.

(19) Jakubczyk P, Napiorkowski M. (2004) The influence of droplet size on line tension. J. Phys.: Cond. Matt. 166917.

(20) Parr A. Hydraulics and Pneumatics: A Technician's and Engineer's Guide, Third Edition. Oxford, U.K.: Butterworth-Heinemann; 2011.

(21) Waugh DG, Lawrence J. The enhancement of biomimetic apatite coatings by means of $\mathrm{KrF}$ excimer laser surface treatment of nylon 6,6. Lasers Eng 21 95114.

(22) Van Oss C.J., Gillman C.F., Neumann A.W. Phagocytic Engulfment and Cell Adhesiveness. New York, USA: Marcel Dekker; 1975.

(23) Renke-Gluszko M., Fray M.E. (2004) The effect of simulated body fluid on the mechanical properties of multiblock poly(aliphatic/aromatic-ester)

copolymers. Biomaterials 25 5191-5198.

(24) Waugh D.G., Lawrence J., Morgan D.J., Thomas C.L. (2009) Interaction of $\mathrm{CO}_{2}$ laser-modified nylon with osteoblast cells in relation to wettability. Mater. Sci. Eng. C 29 2514-2524.

(25) Waugh D.G., Lawrence J. (2011) Wettability and osteoblast cell response modulation through UV laser processing of nylon 6,6. App. Surf. Sci. 257 87988812.

\section{Meet the Author}

Dr. David Waugh completed his Ph.D. under the supervision of Dr. Jonathan Lawrence in July 2010. Dr. Waugh is now a Lecturer at the newly established School of Engineering at the University of Lincoln, UK. He is also part of the Lincoln Laser Engineering Group in which he primarily focuses on the application of laser surface treatment to life sciences. He obtained his MPhys Hons. in Physics with Lasers and Photonics and MSc in Laser Applications in Micro-Machining and Processing from the University of Hull, UK. 